\title{
Microalgae: therapeutic potentials and applications
}

\author{
Fatemeh Khavari $^{1,2} \cdot$ Massoud Saidijam $^{2}\left(\right.$ Mohammad Taheri $^{3}\left[\right.$ Matemeh Nouri $^{4}([)$
}

Received: 8 March 2021 / Accepted: 17 May 2021 / Published online: 24 May 2021

(c) The Author(s), under exclusive licence to Springer Nature B.V. 2021

\begin{abstract}
Recently, special attention has been paid to marine origin compounds such as carbohydrates, peptides, lipids, and carotenoids, which are extracted from microalgae and have anticancer, anti-inflammatory, antimicrobial (e.g., anti-COVID-19 activity), and antioxidant properties in biomedicine and pharmaceutical biotechnology. In addition, these photosynthetic marine microorganisms have several applications in biotechnology and are suitable hosts for the production of recombinant proteins/peptides, such as monoclonal antibodies and vaccines. Silica-based nanoparticles obtained from diatoms (a microalgae group) are used as drug delivery carriers owing to their biodegradability, easy functionalization, low cost, and simple features compared to synthetics, which make these agents proper alternatives for synthetic silica nanoparticles. Therefore, diatom-based nanoparticles are a viable option for the delivery of anti-cancer drugs and reducing the side-effects of cancer chemotherapy.
\end{abstract}

Keywords Microalgae $\cdot$ Therapeutic $\cdot$ Drug delivery

\section{Introduction}

Microalgae are photosynthetic microorganisms, which are classified into two categories of prokaryotes and eukaryotes. Prokaryotic microalgae such as cyanobacteria are known as blue-green algae, and eukaryotic microalgae comprise diatom and green algae. These microorganisms could adapt to mild and harsh conditions and survive in different environments [1-5]. Microalgae are capable of producing various types of bioactive molecules, including carotenoids, polysaccharides, vitamins, and lipids. Furthermore, they have potential uses as antioxidant, anti-inflammatory, antitumor,

Fatemeh Nouri

Fatemenouri1@gmail.com

Fatemeh Khavari

khavari.fateme@edu.umsha.ac.ir

1 Student Research Committee, Hamadan University of Medical Sciences, Hamadan, Iran

2 Research Center for Molecular Medicine, Hamadan University of Medical Sciences, Hamadan, Iran

3 Department of Medical Microbiology, Faculty of Medicine, Hamadan University of Medical Sciences, Hamadan, Iran

4 Department of Pharmaceutical Biotechnology, School of Pharmacy, Hamadan University of Medical Sciences, Hamadan, Iran anticancer, antimicrobial, antiviral, and anti-allergy agents; therefore, they play a key role in the biomedical and pharmaceutical industry. Microalgae are also applied in cosmetic products, environmental biotechnology, and animal feeds [1, 6-8].

In the past decade, microalgae-based nanoparticles have been used in drug delivery systems. The main advantages of these nanoparticles over other carriers are low toxicity, biodegradability, and large surface area. Microalgae play a pivotal role in medical and pharmaceutical biotechnology and are used for the synthesis of growth factors, hormones, antibodies, vaccines, and immune regulators $[9,10]$.

Today, cancer is considered to be a leading cause of death across the world [11]. Current cancer treatments are surgery, radiotherapy, and chemotherapy. One of the most important challenges of chemotherapy is the associated complications since in addition to tumor cells, chemotherapy drugs also target non-tumor cells in the body. These complications could be minimized by drug delivery systems and the specific targeting of tumor cells [12].

Diatom is a eukaryotic unicellular microalgae with a unique cell wall known as frustule. Frustule structure is composed of silica and has notable properties for drug delivery, such as highly-ordered three-dimensional (3D) pores, chemical inertness, microchannel, and uniform nanopore structure. Furthermore, frustules could be easily protected, 
functionalized, and engineered for successive drug loading and delivery [13, 14] (Fig. 1).

\section{Applications of microalgae}

\section{Food and human health}

Microalgae such as Spirulina and Chlorella are consumed as food and dietary supplements owing to their high nutritional value. Microalgae are an important source of proteins, essential amino acids, carbohydrates (e.g., glucose and starch), ๑-3 and $\omega-6$ fatty acids, and vitamins B1, B2, B5, B6, B9, A, C, and E. Spirulina has a high content of vitamins, minerals, proteins, and $\gamma$-linolenic acid and has shown therapeutic effects on various health issues, such as arthritis, diabetes, cardiovascular diseases, and cancer.

Chlorella is another important microalgae in the food industry, which has a high content of protein, carotenoids, vitamins, and $\beta$-glucan (free radical scavenger and immune system stimulator). Similar to Spirulina, Chlorella and Dunaliella are used in the food and pharmaceutical industry, with an annual global income worth hundreds of thousands of dollars $[9,14]$.

\section{Polyunsaturated fatty acids}

Eicosapentaenoic acid and docosahexaenoic acid are the most important $\omega-3$ fatty acids in microalgae, which largely benefit the development of the eyes and brain in infants, while also contributing to the prevention of cardiovascular diseases, blood coagulation, nervous system functions, and blood pressure maintenance in adults. The microalgae that produce these lipids are Haematococcus, Spirulina, Schizachyrium, and Crypthecodinium [14-16].

\section{Carbohydrates}

Cyanobacteria are a major source of exocellular polysaccharides, which are used as stabilizers in the food industry and as hydrating agents in cosmetics and pharmaceuticals. Some microalgae polysaccharides are also used in lotions and creams given their antioxidant properties. Moreover, sulfated polysaccharides extracted from microalgae stimulate the human
Fig. 1 Graphical abstract of the presented study

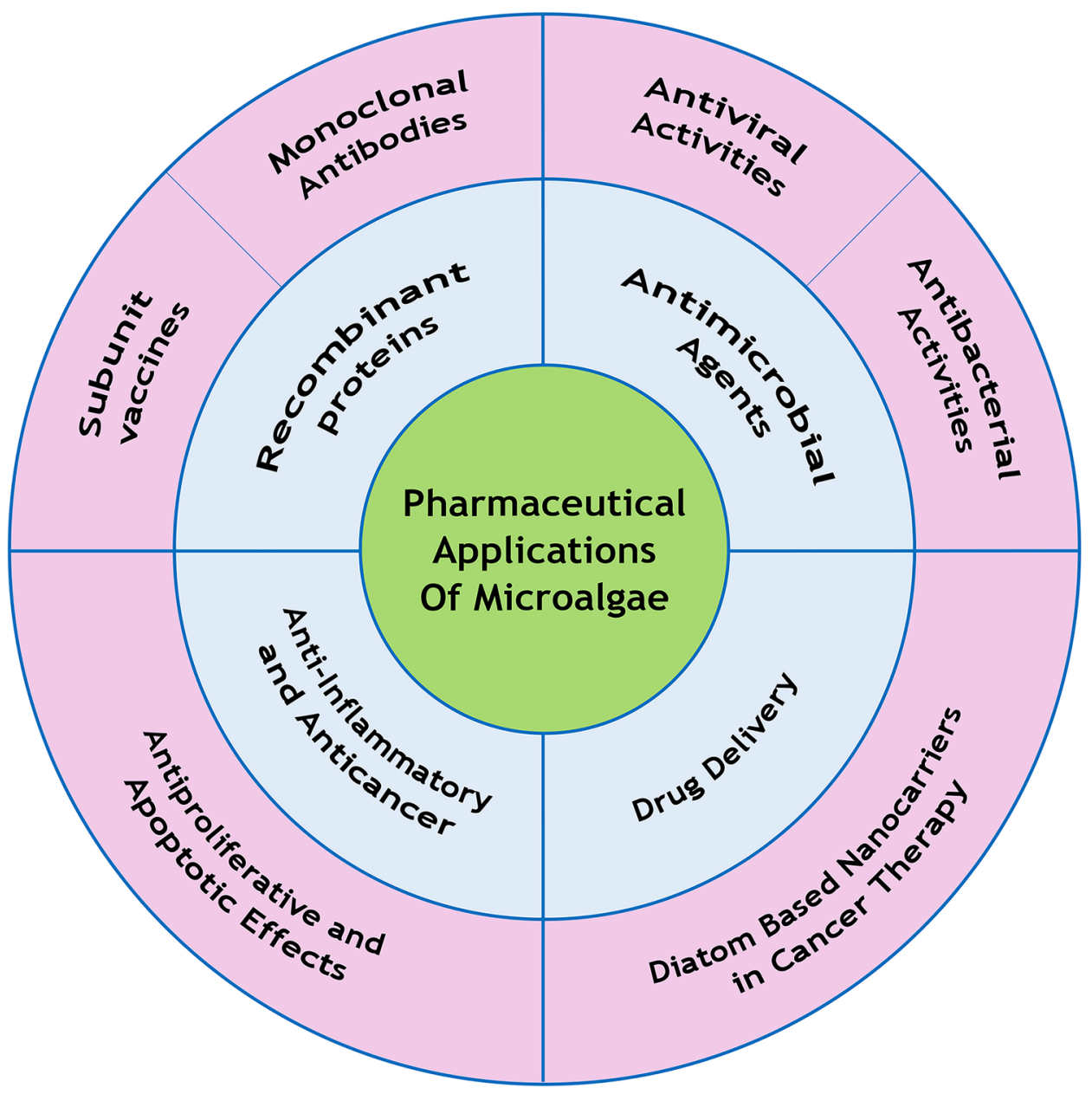


immune system. In-vivo and in-vitro studies have indicated that the polysaccharides of some microalgae affect the herpes simplex virus [16].

\section{Amino acids, peptides, and proteins}

Spirulina and Chlorella vulgaris have significant protein content. Proteins of Spirulina could reduce inflammation and allergies, and peptides of $C$. vulgaris could prevent cell damage. Furthermore, microalgae contain essential amino acids that cannot be synthesized by mammals $[15,17]$.

\section{Carotenoids}

Carotenoids are the pigments polymerized from isoprene units. Beta-carotene, astaxanthin, lutein, zeaxanthin, and lycopene are important carotenoids of microalgae. Beta-carotene is used in pharmaceutical industries owing to its antioxidant activity, which prevents cancer. Astaxanthin is another carotenoid in microalgae and a scavenger of free radicals, which has notable properties such as skin protection, immune function improvement, and protection against cancer and inflammatory diseases $[14,16,18]$.

\section{Pharmaceutical applications}

Microalgae are capable of producing bioactive compounds such as antibiotics, subunit vaccines, monoclonal antibodies, hepatotoxic and neurotoxic compounds, hormones, enzymes, and other compounds with pharmaceutical and therapeutic applications, which are not easily synthesized by chemical methods [8]. Microalgae pigments also have health benefits such as the prevention of cancer, cardiac diseases, neurological disorders, and eye diseases. Microalgae are an ideal host for the production of recombinant proteins given their unique properties, such as the rapid growth rate and simple and lowcost media, while their post-translational modifications are rather similar to mammalian cells as opposed to bacterial cells [19].

Chlamydomonas reinhardtii is the most popular microalgae in pharmaceutical biotechnology. Antibodies [19], vaccines, erythropoietin, and viral protein 28 (VP 28) are the recombinant proteins produced in this microalgae [9]. Chlorella, Dunaliella, and Scenedesmus are other species that could produce recombinant proteins. For instance, $D$. salina produces HBsAg and VP 28 in the nucleus and $\alpha$-galactosidase and phytase in the chloroplast $[9,20,21]$.

\section{Production of pharmaceuticals and therapeutic proteins}

\section{Subunit vaccines}

Microalgae (especially $C$. reinhardtii) are viable options to be used as vaccine transporters as they are safe and contain a single chloroplast, which expresses proteins with high accumulation. Such examples of these recombinant proteins are E2 protein, which is used in the vaccines against classical swine fever virus, D2-CTB fusion protein (D2 fibronectinbinding domain of $S$. aureus containing cholera toxin B subunit), which is used in the oral vaccine against $S$. aureus, and E7 oncoprotein, which is applied in the human papillomavirus (HPV) vaccines [9, 22]. In a study in this regard, Dermutas et al. inserted the gene of HPV16 E7 protein into the genome of $C$. reinhardtii chloroplast, which expressed E7GGG protein for therapeutic vaccines. Therefore, the microalgae was reported to be a proper alternative of plant hosts [23].

\section{Monoclonal antibodies}

Today, most monoclonal antibodies are produced in Chinese hamster ovary cell lines and are highly costly and associated with the risk of contamination with human pathogens. Owing to their benefits, microalgae are considered to be effective alternative host cells. These eukaryotic microorganisms are superior to bacterial cells in the post-translational modifications of human recombinant proteins. Furthermore, they are preferred to other eukaryotic hosts given their high growth rate, convenient handling, and simple culturing. In a research, Hampel et al. produced a monoclonal IgG antibody in engineered Phaeodactylum tricornutum against the nucleoprotein of Marburg virus, which is a leading cause of hemorrhagic fever in western Africa [24, 25].

\section{Antimicrobial agents}

Several microalgae extracts have antiviral, antibacterial, antifungal, and antiprotozoal properties. Such examples are indoles, phenols, fatty acids, and volatile halogenated hydrocarbons. In addition, blue-green algae such as Ochromonas sp. and Prymnesium parvum produce toxins with pharmaceutical applications [8].

\section{Antiviral activities}

In general, viral infections are divided into three stages. The first stage involves attachment and the penetration of the virus into the host cell, the second stage is the replication 
of the virus in the host cell, and stage three is the release of the virus particles from the cell. The antiviral compounds of microalgae affect different stages of viral infections. For instance, sulfated polysaccharides interfere with the first stage [2]. In a study in this regard, Hayashi et al. could extract a monogalactosyl diacylglycerol (MGDG) from Coccomyxa sp., which caused physical changes in HPV to envelop. As a result, the virus could not attach to the host cell, indicating the antiviral effects of MGDG [26].

\section{Antibacterial activities}

Today, antibiotic resistance is considered to be a significant challenge in the treatment of infectious diseases. Therefore, it is essential to discover novel antibiotics. In a study, Pratt et al. produced a mixture of fatty acids from Chlorella, which exerted inhibitory effects on bacteria. It seems that the free fatty acids extracted from microalgae are able to kill or inhibit the growth of different gram-positive and gramnegative bacteria. In addition to the antibacterial activity of microalgae, biocompounds have exhibited antibiofilm activities, which play a key role in the treatment of infectious diseases. For instance, Streptococcus mutans and Lactobacillus sp. are two bacteria that form biofilm on teeth and cause the formation of dental plaque. C. vulgaris and D. salina extracts could inhibit biofilm formation and prevent dental caries [2, 27].

According to the study by Santoyo et al., the short-chain fatty acids extracted from Haematococcus pelvis have antibacterial activities against $E$. coli and $S$. aureus, and the solvent used for their extraction could affect the intensity of their antibacterial activities [28]. In this regard, Bhagavathy et al. reported that Chlorococcum humicola extracts exerted different antibacterial effects on six bacteria. In the mentioned study, this antibiotic activity was associated with the presence of phenol compounds and pigments such as $\beta$-carotene and chlorophyll II, which are the pigments extracted from Chlorococcum humicola with antibacterial effects against Vibrio cholera, Salmonella typhimurium, $S$. aureus, and Bacillus subtilis [2, 18, 28, 29].

\section{Anti-inflammatory and anticancer properties}

Some carbohydrates, lipids, and phycobiliproteins that are extracted from microalgae have shown antiproliferative and apoptotic effects on various cancers. Fucoidan is a sulfated polysaccharide, which is extracted from different microalgae, such as Fucus vesiculosus, Sargassum henslowianam, Cladosiphon fucoidan, and Coccophora longsdorfi, which inhibit angiogenesis and metastasis through the down-regulation of kinase activity and activation of caspase-3/7 in the human lymphoma cell line, melanoma, human colon cancer, breast cancer, lung carcinoma, and human promyeloid leukemia [18].

In a research in this regard, Miceliet al. reported that the monoacylglycerides extracted from Skeletonema marinoi could induce selective apoptosis through the activation of caspase-3/7 in the colon cancer cell line (HCT-116) and hematological cancer cell line (U-937), while no apoptosis was induced in normal cells [30]. Furthermore, some microalgae lipids (e.g., polyunsaturated fatty acids) have shown antitumor activities against cervical and breast cancer. Phycocyanin is a phycobiliprotein extracted from microalgae such as Arthronema africanum, Spirulina platensis, and Porphyra haitanensis, which inhibit the growth of human hepatocellular carcinoma, lung/colon cancer, and leukemia cells [18].

\section{Microalgae and COVID-19 treatment}

Currently, coronavirus disease (COVID-19) is the most important health issue across the world, and there is an urgent need for finding effective treatments for this disease and preventing hundreds of thousands of deaths [31]. Studies have indicated that the acute respiratory distress syndrome (ARDS) resulted from the cytokine storm syndrome is a major cause of death in COVID-19 patients. In cytokine storm, the level of pro-inflammatory cytokines (IL-1, IL-6, TNF- $\alpha$ ) and chemokines (CCL2, CCL3, CXCL10, CXCL9) increase, thereby causing the hyperactivity of the immune system and acute lung injury (ALI) [32].

Astaxanthin is a carotenoid with anti-inflammatory, immunomodulatory, and antioxidative properties, as well as other therapeutic activities. Haematococcus pluvialis is a microalgae and a natural source of astaxanthin. Studies have indicated that the administration of this carotenoid to COVID-19 patients could alleviate cytokine storm, thereby preventing ARDS and ALI [31].

Lectins are proteins that attach to specific mono and oligosaccharides. Cyanovirin-N is a lectin extracted from cyanobacteria (Nostoc ellipsosporum), which has exhibited anti-viral activities against HIV, influenza, and the Ebola virus. Carrageenan is a sulfated polysaccharide of microalgae origin, which is able to inhibit the attachment, transcription, and replication of viruses in host cells [33]. In a study, Koenighofer et al. produced a nasal spray containing zanamivir (antiviral drug), and carrageenan was reported to exert synergistic effects on the influenza virus [34].

Spirulina is a blue-green microalgae with high protein content, several vitamins, $\gamma$ - linolenic acid, and tocopherol. In addition, Spirulina has nutritional and therapeutic applications and is referred to as a 'superfood'. Studies have indicated that these microalgae have potent antiviral activities. Calcium spirulan is a polysaccharide derived from spirulina, 
which inhibits the replication of several viruses, such as influenza, mumps, and HIV [35]. Furthermore, S. platensis could activate the immune system against viruses through the activation of immune cells and inducing the production of interferon-gamma, which is an important cytokine with antiviral activity. Phycocyanin is also a pigment obtained from spirulina and an inhibitor of NADPH oxidase with antiinflammatory activity. It seems that microalgae (especially Spirulina) are effective candidates for the adjuvant therapy of COVID-19 patients [35].

\section{Microalgal-based micro-/nanocarriers in drug delivery}

Drug delivery systems are designed to target drugs or genes to the target cells, such as cancer cells. Patients with genetic disorders commonly have a defective or absent genome. Gene delivery with silica nanoparticles (NPs) is considered to be an effective approach in this regard [36]. Today, advanced drug delivery systems could overcome the limitations of conventional drugs (e.g., poor solubility/stability and high toxicity) as they carry drugs to specific tissues in the body.

Liposomes, micelles, and silicon oxide NPs are among commonly used drug carriers, which have advantages and limitations. Silica-based NPs such as MCM-41 and SBA-15 have unique physical and chemical properties, such as a high surface area, tailorable pore size, thermal ability, and high loading capacity. As for the limitations of these NPs, it has been reported that their synthesis is costly and time-consuming and requires high energy and toxic materials [10,37].

Today, emphasis is placed on the use of marine resources in biomedicine [38]. In the past decade, diatom biotechnology attracted great attention as an important area for the research and production of high-value compounds with pharmaceutical applications [39]. As mentioned earlier, microalgae are major sources of various polysaccharides, such as alginate, carrageenan, laminarin, and fucoidan, which could be converted into NPs and use hydrophilic groups on the surface to interact with biomolecules [40].

Diatoms are brown algae with an amorphous silica exoskeleton, which have easy and costly cultivation. Their fossils [diatomaceous earth (DE)/frustules] are a source of porous silica $\left(\mathrm{SiO}_{2}\right)$ NPs. Live diatoms and DE are considered to be sources of silica NPs, while DE contains more quantities of frustules [37, 41]. In addition, silica NPs modified with cationic reagents (e.g., cationic amino silanes) are used for gene delivery.

Functionalization with amino groups protects plasmid DNA and delivers DNA to the nucleus. In a research in this regard, Park et al. reported that receptor-mediated endocytosis increased transfection efficacy and decreased cytotoxicity
[42]. Furthermore, Aw et al. investigated the oral delivery of a hydrophobic drug (indomethacin) and a hydrophilic drug (gentamicin) through porous silica diatoms and observed that indomethacin loading (smaller than gentamicin) was higher and had stronger interaction with DE, while the drug release behavior of both drugs was similar [43]. In another study, Bariana et al. used organosilanes and phosphonic acid for modification of diatoms to use for co-delivery of hydrophobic (indomethacin) and hydrophilic (gentamicin) drugs. They found hydrophilic modifications such as 16-PHA (16-phosphono-hexadecanoic acid), and OTS (7-octadecyltrichlorosilane) improved drug loading and controlled release of gentamicin, whereas hydrophilic modifications such as APTES (3-aminopropyltriethoxysilane) and 2-CEPA (2-carboxyethyl-phosphonic acid) enhanced drug loading and controlled release of indomethacin [44].

Modifications could alter and improve the properties of diatomaceous earth (Diatomite; DE); for instance, the oligo(ethylene glycol) methacrylate copolymers on diatom microcapsules render these agents stimulus-responsive carriers for the delivery of levofloxacin, which is an antibiotic used against $S$. aureus and Pseudomonas aeruginosa [45]. Moreover, diatom frustules modified with DOPA/ $\mathrm{Fe}_{3} \mathrm{O}_{4}$ (dopamine-terminated $\mathrm{Fe}_{3} \mathrm{O}_{4} \mathrm{NPs}$ ) could control an external magnetic field through their magnetic properties [46]. In research in this regard, Sasirokha et al. performed the chitosan functionalization of Amphora subtropica for doxorubicin (DOX) loading and release [47]. In addition, Janićijević et al. reported that diatomite modified with aluminum sulfate is an effective carrier for diclofenac sodium, providing higher adsorbent loading and extended release compared to control [48]. In another study, cargo molecules in biomimetic silica carriers (e.g., drugs, bioactive peptides/proteins) were conjugated with R5 silaffin peptide via a disulfide linkage. Following that, R5-cargo conjugates were entrapped in silica particles, and acidic and reductive conditions were observed to be suitable for the release of cargo from this complex [49].

Gnanomoothy et al. used natural silica NPs derived from Coscinodiscus concinnus (diatom) for the delivery of streptomycin (hydrophilic drug), observing that the drug release efficiency time of the treated diatom was extended compared to the untreated diatoms. In addition, streptomycin was adsorbed inside the pores and into the hallow diatom structure due to surface adsorption [50].

According to the study by Vona et al., increasing silanol at the surface of diatom increased the drug encapsulation of ophiobolin A, which is an anticancer molecule derived from fungi. In the mentioned study, surface functionalization was reported to be an effective measure for drug loading on diatoms, and ophiobolin A release was observed to be extended $[13,51]$. In the study conducted by Cicco et al., nanoporous silica-based particles obtained from Thalassiosira weissflogii 
were functionalized with cyclic nitric oxide 2,6,6-tetramethyl piperidine-N-oxyl and loaded with an antibiotic (ciprofloxacin), exerting antioxidant and antibacterial effects on fibroblast and osteoblast-like cell growth [52].

Bariana et al. investigated the combined effects of the surface functionalization and morphology of diatoms on their drug loading and release properties [44]. Diclofenac sodium(DS) is a non-steroidal anti-inflammatory drug(NSAID) that uses in painful and inflammation conditions. This drug drawback is short biological half-life and high metabolize rate in the liver, so it should be administered frequently. diatomaceous earth(DE) particles modified with xerogel could prolong and control the release of DS so are suitable carriers for this drug [53].

\section{Diatom-based nanocarriers in cancer therapy}

Cancer is the cause of 171.2 of 100,000 deaths each year. Common anti-cancer drugs inhibit DNA synthesis and have antimetabolite activities. However, a major challenge in cancer therapy is the impact of anticancer drugs on normal cells and tissues. NPs are considered to be effective carriers for the specific delivery of drugs to tumor cells. Using synthetic NPs for the delivery of anticancer drugs is an important approach to the improvement of chemotherapy effectiveness. However, these materials may be toxic and have some environmental disadvantages. In recent decades, the use of biodegradable silicon NPs (SiNPs) has been replaced with microalgae, which have cost-efficient manufacturing and low toxicity. In a study, Maher et al. used the gas-solid displacement method for the conversion of frustules into SiNPs, while also using SiNPs loaded with DOX for cancer therapy. The findings of the mentioned study indicated that the cytotoxic effects of DOX-SiNPs were superior to free DOX [54].

Several compounds derived from microalgae have shown anti-cancer properties and are a viable option for cancer therapy. Nevertheless, natural compound-based anticancer drugs (e.g., camptothecin) have poor water solubility. The encapsulation of camptothecin into NPs has been reported to enhance its solubility, increase its half-life in the bloodstream, and lead to the better accumulation of the drug in cancer tissues.

Bioactive compounds of blue-green algae can induce apoptosis and activate the protein kinase-c family in cancer cells [55]. Using drug delivery systems is considered to be an effective approach to the reduction of toxicity and improving the therapeutic efficacy of drugs, especially in cancer therapy [56]. As mentioned earlier, diatomite silica NPs (DSNs) are a proper candidate for cancer therapy. DSNs are also efficient nanocarriers for the uptake of anticancer molecules into the cytoplasm of human epidermoid carcinoma cells [57]. Currently, delivery carriers such as liposomes and polymeric nanoparticles [58] are applied in chemotherapy, and two or more drugs may be used as dual drug delivery. For instance, DOX and paclitaxel (PTX) have exhibited synergistic interactions in the treatment of solid tumors when the concentration of DOX is higher than PTX. Notably, DE particles are appropriate for the controlled release of these drugs given their easy synthesis and self-assembly [59]. Terracciano et al. used biofunctionalized diatom nanoparticles (DNPs) for drug loading and release studies of sorafenib, a poorly water-soluble anticancer drug. They found DNPs functionalized with ATPES(3-Aminopropyl) triethoxysilane and polyethylene glycol (PEG) showed excellent cellular uptake and better-sustained drug release profile [60]. Curcumin has antioxidant, anti-inflammatory, and anticancer properties. Meanwhile, using curcumin for therapeutic purposes is associated with limitations such as rapid dimeration, low bioavailability, and poor absorption. Natural diatoms modified with polydopamine are efficient carriers for the delivery of curcumin in cancer therapy [61]. Moreover, diatomites activated by oxidizing acids have been used as a carrier of ophiobolin A (anticancer compound of fungal origin), extending the release of this agent [13]. According to the research by Todd et al., diatoms loaded with iron oxide NPs could be used as smart carriers for the delivery of small molecules and drugs by controlling an external magnetic field with their magnetic properties [62]. At the specific $\mathrm{pH}$ of the gastrointestinal (GI) tract, this complex attaches to HCT- 116 colorectal cancer cells, and the anticancer drug is slowly released under light irradiation, thereby increasing cytotoxicity against HCT-116 two-fold [63]. On the other hand, free diatoms have shown extremely low cytotoxicity against Caco-2, HT-29, and HT-116 (colon cancer cells), thereby enhancing the sustained release and permeation of mesalamine and prednisone in the GI tract [64].

Diatom silica microparticles have been used for the delivery of two drugs in the treatment of GI diseases. Mesalamine and prednisone have a prolonged release in GI disorders. In addition, drug permeability across Caco-2/HI-29 cells has been reported to improve, and these particles are regarded as proper candidates for colon cancer treatment [64]. Diatomite NPs are also effective in the transport of siRNA inside human epidermoid cancer cells (H1355) to silence gene expression. These nanocarriers could also be loaded with one or more different molecules and improve the delivery of antitumor biomolecules and drugs [65].

Scytonemin regulates the formation of kinase and controls the cell cycle; therefore, it has inhibitory activity against human endothelial cells. Calothrixin B is a product of calothrix and has antiproliferative activity against HCT-11 colon cancer cells. Calothrixin B is obtained from Calothrix, malyngamides is derived from Lyngbya majuscula, merocyclophanes A and B are obtained from 
Nostoc sp., and hierridin B is derived from Cyanobacterium sp., all of which have cytotoxic effects against colon cancer cells. Lutein (polysaccharide extracted from $C$. vulgaris) and violaxanthin derived from Chlorella ellipsoidea have shown anti-proliferative activity against HCT-116 cells [11]. In a study in this regard, Ragendran et al. used Amphora subtropica functionalized with chitosan for the delivery of DOX, reporting the improvement of drug delivery to cancer cells. Furthermore, cytotoxic effects were observed to enhance compared to free DOX due to the sustained drug release from Chi@AF-DOX [47] (Table 1).

\section{Conclusion}

High-value products have been derived from microalgae with numerous health and pharmaceutical applications. Microalgae are microorganisms and biorefinery factories for the production of recombinant proteins. In addition, diatom shells have unique 3D structures and are used for the production of NPs for drug and biomolecule delivery. Different morphology and functionalization could enhance drug loading and release from DE NPs. Several studies have investigated modified DEMPs for the specific delivery of drugs (DOX, camptothecin, paclitaxel) in the treatment of colon and breast cancer, proposing positive outcomes. Further investigation and clinical trials are still required in this regard.

Table 1 Various applications of microalgae in health

\begin{tabular}{|c|c|c|c|c|}
\hline Application & Metabolite & Microalgae & Action & Ref. \\
\hline \multirow[t]{4}{*}{$\begin{array}{l}\text { Applications } \\
\text { in food } \\
\text { and human } \\
\text { health }\end{array}$} & $\begin{array}{l}\text { Polyunsaturated fatty acids (PUFA) } \\
\text { (EPA \& DHA) }\end{array}$ & $\begin{array}{l}\text { Haematococcus, Spirulina, Schizachy- } \\
\text { rium, and Crypthecodinium }\end{array}$ & $\begin{array}{l}\text { Prevention of cardiovascular disease } \\
\text { in adults, blood coagulation, func- } \\
\text { tions of nervous system and blood } \\
\text { pressure maintenance }\end{array}$ & {$[14,15]$} \\
\hline & $\begin{array}{l}\text { Carbohydrates (sulfated polysaccha- } \\
\text { rides) }\end{array}$ & Porphyridium cruentum & $\begin{array}{l}\text { Stabilizers in the food industry, } \\
\text { hydrating agents in cosmetics and } \\
\text { pharmaceuticals, stimulate the } \\
\text { human immune system }\end{array}$ & {$[15,16]$} \\
\hline & Amino acids, Peptides, and Proteins & Spirulina and Chlorella vulgaris & Reduce inflammation and allergies & [15] \\
\hline & $\begin{array}{l}\text { Carotenoids ( } \beta \text {-carotene, astaxanthin, } \\
\text { lutein, zeaxanthin, and lycopene) }\end{array}$ & Chlorella & $\begin{array}{l}\text { Antioxidant activity for the prevention } \\
\text { of cancer, skin protection, empower- } \\
\text { ing the immune system }\end{array}$ & {$[14,16]$} \\
\hline \multirow{11}{*}{$\begin{array}{l}\text { Pharmaceu- } \\
\text { tical Appli- } \\
\text { cations }\end{array}$} & \multirow[t]{2}{*}{ Production of therapeutic proteins } & C. reinhardtii & HPV vaccine (E7 oncoprotein) & [9] \\
\hline & & Phaeodactylum tricornutum & $\begin{array}{l}\text { Monoclonal IgG antibody against the } \\
\text { nucleoprotein of Marburg virus }\end{array}$ & {$[24]$} \\
\hline & \multirow[t]{3}{*}{ Antimicrobial agents } & $\begin{array}{l}\text { Coccomyxa sp (monogalactosyl dia- } \\
\text { cylglyceride (MGDG) extract) }\end{array}$ & $\begin{array}{l}\text { Physical changes in human papilloma- } \\
\text { virus (HPV) envelop }\end{array}$ & {$[27]$} \\
\hline & & $\begin{array}{l}\text { Chlorella vulgaris and Dunaliella } \\
\text { salina }\end{array}$ & $\begin{array}{l}\text { Inhibit biofilm formation and prevent } \\
\text { dental caries }\end{array}$ & [27] \\
\hline & & Haematococcus pluvialis & $\begin{array}{l}\text { Antibacterial activity against } E . \text { coli } \\
\text { and } S \text {. aureus }\end{array}$ & {$[28]$} \\
\hline & \multirow[t]{2}{*}{ Anti-cancer activity } & $\begin{array}{l}\text { Fucoidan (Fucus vesiculus, Sargas- } \\
\text { sum henslowianam, Cladosiphon } \\
\text { fucoidan) }\end{array}$ & Inhibits angiogenesis and metastasis & {$[18]$} \\
\hline & & $\begin{array}{l}\text { Monoacylglycerides (MAGs) (skel- } \\
\text { etonema marinoi) }\end{array}$ & $\begin{array}{l}\text { Induce selective apoptosis through } \\
\text { activation of caspase } 3 / 7\end{array}$ & {$[30]$} \\
\hline & \multirow[t]{4}{*}{ Drug delivery } & $\begin{array}{l}\text { Amorpha subtropica, which function- } \\
\text { alized with chitosan (chi) }\end{array}$ & $\begin{array}{l}\text { Enhancing doxorubicin delivery to } \\
\text { cancer cells }\end{array}$ & {$[63]$} \\
\hline & & $\begin{array}{l}\text { Natural silica nanoparticles from Cos- } \\
\text { cinodiscus concinnus (a diatom) }\end{array}$ & Improving streptomycin delivery time & [49] \\
\hline & & $\begin{array}{l}\text { Nanoporous silica-based particles } \\
\text { from Thalassiosira weissflagii, } \\
\text { which functionalized with TEMPO }\end{array}$ & $\begin{array}{l}\text { Enhancing antioxidant and antibacte- } \\
\text { rial activity of ciprofloxacin }\end{array}$ & {$[45]$} \\
\hline & & $\begin{array}{l}\text { Natural diatoms modified with polydo- } \\
\text { pamine (PDA) }\end{array}$ & $\begin{array}{l}\text { Suitable carriers for the delivery of } \\
\text { curcumin with the aim of cancer } \\
\text { therapy }\end{array}$ & {$[61]$} \\
\hline
\end{tabular}


Acknowledgements The authors would like to acknowledge the Vicechancellor of Research and Technology, Hamadan University of Medical Sciences, Hamadan, Iran.

Author contributions All authors have an equal role in writing and approved the final manuscript.

Funding The Vice-chancellor of Research and Technology, Hamadan University of Medical Sciences, Hamadan, Iran, financially supported the study (Grant No. 9910307639).

Data availability The datasets used and/or analyzed during the current study available from the corresponding author on reasonable request.

\section{Declarations}

Conflict of interest The authors declare that they have no competing interest.

Ethical approval This study was approved by the ethics committee of Hamadan University of Medical Sciences (IR.UMSHA. REC.1399.818)

\section{References}

1. Fu W et al (2019) Advances in microalgal research and engineering development. Curr Opin Biotechnol 59:157-164

2. Mostafa SS (2012) Microalgal biotechnology: prospects and applications. Plant Sci 12:276-314

3. Ghasemi Y et al (2009) C-20 ketone reduction of hydrocortisone by rice field microalga Chlorella vulgaris MCCS 013. Chem Nat Compd 45(6):824-828

4. Rasoul-Amini S et al (2010) Characterization of hydrocortisone bioconversion and 16S RNA gene in Synechococcus nidulans cultures. Appl Biochem Microbiol 46(2):191-197

5. Ghasemi Y et al (2008) Characterization of hydrocortisone biometabolites and 18S rRNA gene in Chlamydomonas reinhardtii cultures. Molecules 13(10):2416-2425

6. Renuka N et al (2016) Exploring the efficacy of wastewater-grown microalgal biomass as a biofertilizer for wheat. Environ Sci Pollut Res Int 23(7):6608-6620

7. Gonzalez-Fernandez C, Muñoz RJK (2017) Microalgae-based biofuels and bioproducts. Woodhead Publishing, UK

8. Rizwan $\mathrm{M}$ et al (2018) Exploring the potential of microalgae for new biotechnology applications and beyond: a review. Renew Sustain Energy Rev 92:394-404

9. Yan $\mathrm{N}$ et al (2016) The potential for microalgae as bioreactors to produce pharmaceuticals. Int J Mol Sci 17(6):962

10. Aw MS et al (2012) Porous silica microshells from diatoms as biocarrier for drug delivery applications. Powder Technol 223:52-58

11. Bajpai VK et al (2018) Developments of cyanobacteria for nanomarine drugs: relevance of nanoformulations in cancer therapies. Mar Drugs 16(6): 179

12. Huang C-Y et al (2017) A review on the effects of current chemotherapy drugs and natural agents in treating non-small cell lung cancer. Biomedicine 7(4):23

13. Vona $D$ et al (2016) Diatoms biosilica as efficient drug-delivery system. MRS Adv. https://doi.org/10.1557/adv.2015.22

14. Chu W-LJI (2012) Biotechnological applications of microalgae. leJSME 6(1):S24-S37
15. Barkia I, Saari N, Manning SR (2019) Manning, microalgae for high-value products towards human health and nutrition. Mar Drugs 17(5):304

16. Hamed I (2016) The evolution and versatility of microalgal biotechnology: a review. Compr Rev Food Sci Food Saf 15(6):1104-1123

17. Lordan S, Ross RP, Stanton C (2011) Marine bioactives as functional food ingredients: potential to reduce the incidence of chronic diseases. Mar Drugs 9(6):1056-1100

18. Deniz I, García-Vaquero M, Imamoglu E (2017) Trends in red biotechnology: microalgae for pharmaceutical applications. Microalgae-based biofuels and bioproducts. Elsevier, Amsterdam, pp $429-460$

19. Ramana KV, Xavier JR, Sharma RK (2017) Recent trends in pharmaceutical biotechnology. Pharm Biotechnol Curr Res 1(1):5

20. Georgianna DR et al (2013) Production of recombinant enzymes in the marine alga Dunaliella tertiolecta. Algal Res 2(1):2-9

21. Geng D et al (2003) Stable expression of hepatitis B surface antigen gene in Dunaliella salina (Chlorophyta). J Appl Phycol 15(6):451-456

22. Specht EA, Mayfield SP (2014) Algae-based oral recombinant vaccines. Front Microbiol 5:60

23. Demurtas OC et al (2013) A chlamydomonas-derived human papillomavirus $16 \mathrm{E} 7$ vaccine induces specific tumor protection. PLoS ONE 8(4):e61473

24. Hempel $\mathrm{F}$ et al (2017) From hybridomas to a robust microalgalbased production platform: molecular design of a diatom secreting monoclonal antibodies directed against the Marburg virus nucleoprotein. Microb Cell Fact 16(1):131

25. Fu W et al (2016) Algal cell factories: approaches, applications, and potentials. Mar Drugs 14(12):225

26. Hayashi $\mathrm{K}$ et al (2019) In vitro and in vivo anti-herpes simplex virus activity of monogalactosyl diacylglyceride from Coccomyxa sp. KJ (IPOD FERM BP-22254), a green microalga. PLoS ONE 14(7):e0219305

27. López Y, Soto SMJA (2020) The usefulness of microalgae compounds for preventing biofilm infections. Antibiotics 9(1):9

28. Santoyo $S$ et al (2009) Green processes based on the extraction with pressurized fluids to obtain potent antimicrobials from Haematococcus pluvialis microalgae. LWT Food Sci Technol 42(7):1213-1218

29. Bhagavathy S, Sumathi P, Bell IJS (2011) Green algae Chlorococcum humicola-a new source of bioactive compounds with antimicrobial activity. Asian Pac J Trop Biomed 1(1):S1-S7

30. Miceli M et al (2019) Monoacylglycerides from the diatom skeletonema marinoi induce selective cell death in cancer cells. Mar Drugs 17(11):625

31. Talukdar J et al (2020) COVID-19: potential of microalgae derived natural astaxanthin as adjunctive supplement in alleviating cytokine storm. SSRN. https://doi.org/10.2139/ssrn.3579738

32. Yang $X$ et al (2020) Clinical course and outcomes of critically ill patients with SARS-CoV-2 pneumonia in Wuhan, China: a singlecentered, retrospective, observational study. Lancet Respir Med. https://doi.org/10.1016/S2213-2600(20)30079-5

33. Rosales-Mendoza S et al (2020) The potential of algal biotechnology to produce antiviral compounds and biopharmaceuticals. Molecules 25(18):4049

34. Koenighofer $\mathrm{M}$ et al (2014) Carrageenan nasal spray in virus confirmed common cold: individual patient data analysis of two randomized controlled trials. Multidiscip Respir Med 9(1):1-12

35. Singh $S$ et al (2020) Therapeutic and nutritional potential of spirulina in combating COVID-19 infection. AIJIR Preprints. https:// doi.org/10.13140/RG.2.2.16527.41127

36. Dolatabadi JEN, de la Guardia M (2011) Applications of diatoms and silica nanotechnology in biosensing, drug and gene delivery, 
and formation of complex metal nanostructures. TrAC Trends Anal Chem 30(9):1538-1548

37. Maher S et al (2018) Diatom silica for biomedical applications: recent progress and advances. Adv Healthcare Mater 7(19): 1800552

38. Chao JT, Biggs MJ, Pandit AS (2014) Diatoms: a biotemplating approach to fabricating drug delivery reservoirs. Expert Opin Drug Deliv. https://doi.org/10.1517/17425247.2014.935336

39. Gordon $\mathrm{R}$ et al (2009) The glass menagerie: diatoms for novel applications in nanotechnology. Trends Biotechnol 27(2):116-127

40. Shankar PD et al (2016) A review on the biosynthesis of metallic nanoparticles (gold and silver) using bio-components of microalgae: formation mechanism and applications. Enzyme Microb Technol 95:28-44

41. Sasirekha R, Santhanam P (2019) Surface bioengineering of diatom by amine and phosphate groups for efficient drug delivery. In: Santhanam P, Begum A, Pachiappan P (eds) Basic and applied phytoplankton biology. Springer, Singapore, pp 229-237

42. Park IY et al (2008) Mannosylated polyethylenimine coupled mesoporous silica nanoparticles for receptor-mediated gene delivery. Int J Pharm 359(1-2):280-287

43. Aw MS et al (2011) Silica microcapsules from diatoms as new carrier for delivery of therapeutics. Nanomedicine 6(7):1159-1173

44. Bariana $\mathrm{M}$ et al (2013) Tuning drug loading and release properties of diatom silica microparticles by surface modifications. Int J Pharm 443(1-2):230-241

45. Vasani R et al (2015) Fabrication of stimulus-responsive diatom biosilica microcapsules for antibiotic drug delivery. J Mater Chem B 3(21):4325-4329

46. Losic D et al (2010) Surface functionalisation of diatoms with dopamine modified iron-oxide nanoparticles: toward magnetically guided drug microcarriers with biologically derived morphologies. Chem Commun 46(34):6323-6325

47. Sasirekha R et al (2019) Surface engineered amphora subtropica frustules using chitosan as a drug delivery platform for anticancer therapy. Mater Sci Eng C 94:56-64

48. Janićijević $\mathrm{J}$ et al (2015) Modified local diatomite as potential functional drug carrier-A model study for diclofenac sodium. Int J Pharm 496(2):466-474

49. Lechner CC, Becker CFW (2013) Modified silaffin R5 peptides enable encapsulation and release of cargo molecules from biomimetic silica particles. Bioorg Med Chem 21(12):3533-3541

50. Gnanamoorthy P, Anandhan S, Prabu VA (2014) Natural nanoporous silica frustules from marine diatom as a biocarrier for drug delivery. J Porous Mater 21(5):789-796

51. Delasoie J, Zobi F (2019) Natural diatom biosilica as microshuttles in drug delivery systems. Pharmaceutics 11(10):537

52. Cicco SR et al (2015) Chemically modified diatoms biosilica for bone cell growth with combined drug-delivery and antioxidant properties. ChemPlusChem 80(7):1104-1112
53. Uthappa U et al (2018) Xerogel modified diatomaceous earth microparticles for controlled drug release studies. New J Chem 42(14):11964-11971

54. Maher $\mathrm{S}$ et al (2016) From the mine to cancer therapy: natural and biodegradable theranostic silicon nanocarriers from diatoms for sustained delivery of chemotherapeutics. Adv Healthcare Mater 5(20):2667-2678

55. Khalifa SA et al (2019) Marine natural products: a source of novel anticancer drugs. Mar Drugs 17(9):491

56. Rigby S, Fairhead M, van der Walle C (2008) Engineering silica particles as oral drug delivery vehicles. Curr Pharm Des 14(18):1821-1831

57. Ruggiero I et al (2014) Diatomite silica nanoparticles for drug delivery. Nanoscale Res Lett 9(1):1-7

58. Nouri F et al (2017) Preparation, characterization, and transfection efficiency of low molecular weight polyethylenimine-based nanoparticles for delivery of the plasmid encoding CD200 gene. Int J Nanomed 12:5557

59. Kabir A et al (2020) Diatoms embedded, self-assembled carriers for dual delivery of chemotherapeutics in cancer cell lines. Int $\mathrm{J}$ Pharm 573:118887

60. Terracciano M et al (2015) Surface bioengineering of diatomite based nanovectors for efficient intracellular uptake and drug delivery. Nanoscale 7(47):20063-20074

61. Uthappa U et al (2019) Facile green synthetic approach of bio inspired polydopamine coated diatoms as a drug vehicle for controlled drug release and active catalyst for dye degradation. Microporous Mesoporous Mater 288:109572

62. Todd $\mathrm{T}$ et al (2014) Iron oxide nanoparticle encapsulated diatoms for magnetic delivery of small molecules to tumors. Nanoscale 6(4):2073-2076

63. Delasoie J et al (2020) Photoactivatable surface-functionalized diatom microalgae for colorectal cancer targeted delivery and enhanced cytotoxicity of anticancer complexes. Pharmaceutics 12(5):480

64. Zhang H et al (2013) Diatom silica microparticles for sustained release and permeation enhancement following oral delivery of prednisone and mesalamine. Biomaterials 34(36):9210-9219

65. Rea I et al (2014) Diatomite biosilica nanocarriers for siRNA transport inside cancer cells. Biochim Biophys Acta Gen Subj 1840(12):3393-3403

Publisher's Note Springer Nature remains neutral with regard to jurisdictional claims in published maps and institutional affiliations. 